\title{
Totally Splittable Polytopes
}

\author{
Sven Herrmann • Michael Joswig
}

Received: 3 January 2009 / Revised: 27 July 2009 / Accepted: 27 July 2009 /

Published online: 19 August 2009

(C) Springer Science+Business Media, LLC 2009

\begin{abstract}
A split of a polytope is a (necessarily regular) subdivision with exactly two maximal cells. A polytope is totally splittable if each triangulation (without additional vertices) is a common refinement of splits. This paper establishes a complete classification of the totally splittable polytopes.
\end{abstract}

\section{Introduction}

Splits (of hypersimplices) first occurred in the work of Bandelt and Dress on decompositions of finite metric spaces with applications to phylogenetics in algorithmic biology [1]. This was later generalized to a result on arbitrary polytopes by Hirai [10] and the authors [9]. While many polytopes do not admit a single split, the purpose of this paper is to study polytopes with very many splits.

The set of all regular subdivisions of a polytope $P$, partially ordered by refinement, has the structure of the face lattice of a polytope, the secondary polytope of $P$ introduced by Gel'fand, Kapranov, and Zelevinsky [6]. The vertices of the secondary polytope correspond to the regular triangulations, while the facets correspond to the regular coarsest subdivisions. There is a host of knowledge on triangulations of polytopes [5], but information on coarsest subdivisions is scarce. Splits are obviously coarsest subdivisions and moreover known to be regular. So they correspond

Sven Herrmann is supported by a Graduate Grant of TU Darmstadt. Research by Michael Joswig is supported by DFG Research Unit "Polyhedral Surfaces".

S. Herrmann · M. Joswig ( $\varangle)$

Fachbereich Mathematik, TU Darmstadt, 64289 Darmstadt, Germany

e-mail: joswig@mathematik.tu-darmstadt.de

S. Herrmann

e-mail: sherrmann@mathematik.tu-darmstadt.de

M. Joswig

Institut für Mathematik, TU Berlin, 10623 Berlin, Germany 
to facets of the secondary polytope. The total splittability of $P$ is equivalent to the property that each facet of the secondary polytope of $P$ arises from a split. Via a compatibility relation the splits of a polytope form an abstract simplicial complex. For instance, for the hypersimplices $\Delta(d, n)$ this turns out to be a subcomplex of the Dressian $\operatorname{Dr}(d, n)$ which is an outer approximation (in terms of matroid decompositions) of the tropical variety arising from the Grassmannian of $d$-planes in $n$-space; see [9, Theorem 7.8] and [8].

As can be expected, the assumption of total splittability restricts the combinatorics of $P$ drastically. We prove that the totally splittable polytopes are the simplices, the polygons, the regular crosspolytopes, the prisms over simplices, or joins of these. Interestingly, our classification seems to coincide with those infinite families of polytopes for which the secondary polytopes are known. This suggests that, in order to derive more detailed information about the secondary polytopes of other polytopes, it is crucial to systematically investigate coarsest subdivisions other than splits. Such a task, however, is beyond the scope of this paper.

This is how our proof (and thus the paper) is organized: It will frequently turn out to be convenient to phrase facts in terms of a Gale dual of a polytope. Hence we begin our paper with a short introduction to Gale duality and chamber complexes. The first important step towards the classification is the easy Proposition 13 which shows that the neighbors of a vertex of a totally splittable polytope must span an affine hyperplane. Then the following observation turns out to be useful: Whenever $P$ is a prism over a $(d-1)$-simplex or a $d$-dimensional regular crosspolytope with $d \geq 3$, there is no place for a point $v$ outside $P$ such that $\operatorname{conv}(P \cup\{v\})$ is totally splittable. In this sense, prisms and crosspolytopes are maximally totally splittable. It is clear that the case of $d=2$ is quite different; and it is one technical difficulty in the proof to intrinsically distinguish between polygons and higher dimensional polytopes. The next step is a careful analysis of the Gale dual of a totally splittable polytope which makes it possible to recognize a potential decomposition as a join. A final reduction argument allows one to concentrate on maximally totally splittable factors, which then can be identified again via their Gale duals.

We are indebted to the anonymous referees for very careful reading which led to several improvements in the exposition.

\section{Splits and Gale Duality}

Let $V$ be a configuration of $n \geq d+1$ (not necessarily distinct) non-zero vectors in $\mathbb{R}^{d+1}$ which linearly spans the whole space. Often we identify $V$ with the $n \times$ $(d+1)$-matrix whose rows are the points in $V$, and our assumption says that the matrix $V$ has full rank $d+1$. Such a vector configuration gives rise to an oriented matroid in the following way: For a linear form $a \in\left(\mathbb{R}^{d+1}\right)^{\star}$ we have a covector $C^{\star} \in\{0,+,-\}^{V}$ by

$$
C^{\star}(v):= \begin{cases}0 & \text { if } a v=0 \\ + & \text { if } a v>0 \\ - & \text { if } a v<0\end{cases}
$$


For $\epsilon \in\{0,+,-\}$ we let $C_{\epsilon}^{\star}:=\left\{v \in V \mid C^{\star}(v)=\epsilon\right\}$, and we call the multiset $C_{+}^{\star} \cup C_{-}^{\star}$ the support of $C^{\star}$. Occasionally, the complement $C_{0}^{\star}$ will be called the cosupport of $C^{\star}$. A covector whose support is minimal with respect to inclusion of multisets is a cocircuit; equivalently, its cosupport is maximal. Dually, $C \subset\{0,+,-\}^{V}$ is called a vector of $V$ if the linear dependence

$$
\sum_{v \in C_{+}} \lambda_{v} v=\sum_{v \in C_{-}} \lambda_{v} v
$$

holds for some coefficients $\lambda_{v}>0$; here $C_{\epsilon}$ is defined as for the cocircuits. The vectors with minimal support are the circuits. Note that a point configuration defines the circuits and cocircuits only up to a sign reversal. Occasionally, we will speak of "unique" (co-)circuits with given properties, and in these cases we always mean uniqueness up to such a reversal of the signs. See monograph [4] for all details and proofs of properties of oriented matroids.

Now consider an $n \times(n-d-1)$-matrix $V^{\star}$ of full rank $n-d-1$ satisfying $V^{\top} V^{\star}=0$; that is, the columns of $V^{\star}$ form a basis of the kernel of $V^{\top}$. Then the configuration of row vectors of $V^{\star}$ is called a Gale dual of $V$. A Gale dual of $V$ is uniquely determined up to affine equivalence. Each vector $v \in V$ corresponds to a row vector $v^{\star}$ of $V^{\star}$, called the vector dual to $v$. Throughout we will assume that all dual vectors are either zero or have unit Euclidean length. If $v^{\star}$ is zero then all vectors other than $v$ span a linear hyperplane not containing $v$. We call $V$ proper if $V^{\star}$ does not contain any zero vectors. In the primal view this means that conv $V$ is not a pyramid. For the remainder of this section, we will assume that $V$ is proper whence $V^{\star}$ can be identified with a configuration of $n$ points on the unit sphere $\mathbb{S}^{n-d-2}$. Notice that these $n$ points are not necessarily pairwise distinct. Repetitions may occur even if the vectors in $V$ are pairwise distinct.

The connection between Gale duality and oriented matroids is the following: The circuits of $V$ are precisely the cocircuits of $V^{\star}$ and conversely. We define the oriented matroid of $V$ as its set of cocircuits. Affinely equivalent vector configurations have the same oriented matroid, but the converse does not hold.

Now let $P$ be a $d$-dimensional polytope in $\mathbb{R}^{d}$ with $n$ vertices. By homogenizing the vertices Vert $P$, we obtain a configuration $V_{P}$ of $n$ non-zero vectors in $\mathbb{R}^{d+1}$ which linearly span the whole space. The cocircuits of $V_{P}$ are given by the linear hyperplanes spanned by vectors in $V_{P}$. The vector configuration $V_{P}$ is proper if and only if $P$ is not a pyramid, and we will assume that this is the case. The Gale dual of $P$ is the spherical point configuration $\operatorname{Gale}(P):=V_{P}^{\star}$, which again is unique up to (spherical) affine equivalence.

Example 1 The matrices

$$
V:=\left(\begin{array}{ccc}
1 & 1 & 0 \\
1 & 0 & 2 \\
1 & -1 & 1 \\
1 & -1 & 0 \\
1 & 0 & -1
\end{array}\right) \quad \text { and } \quad V^{\star}:=\left(\begin{array}{cc}
-1 / 3 & -1 \\
2 / 3 & 1 \\
-4 / 3 & -1 \\
1 & 0 \\
0 & 1
\end{array}\right)
$$



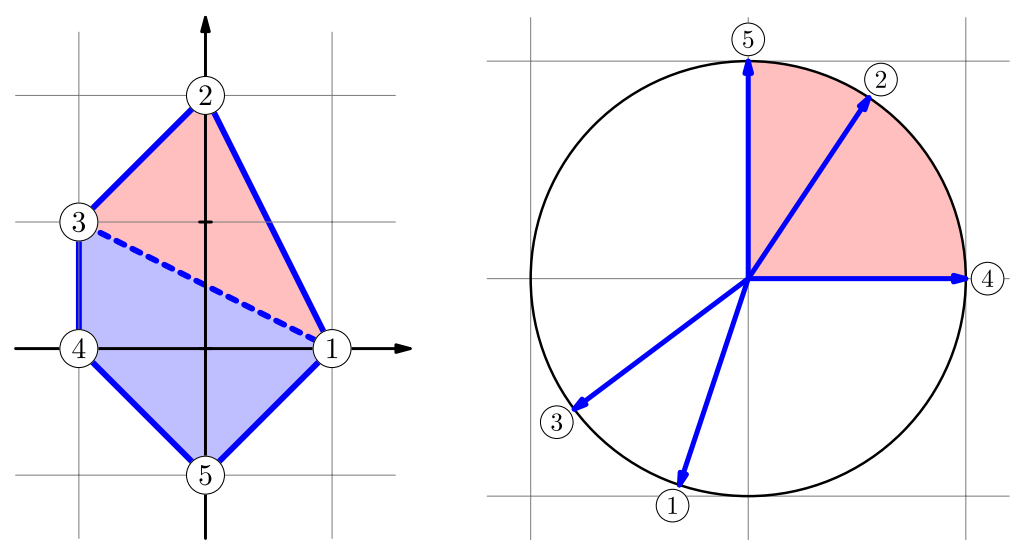

Fig. 1 Pentagon and Gale dual. Corresponding vertices and dual vectors are labeled alike

are Gale duals of each other. The rows of the matrix $V$ are the homogenized vertices of the pentagon shown to the left in Fig. 1. The Gale dual obtained from projecting $V^{\star}$ to $\mathbb{S}^{1}$ is shown to the right.

We are interested in polytopal subdivisions of our polytope $P$ and intend to study them via Gale duality. This requires the introduction of some notation. A polytopal subdivision of $P$ is regular if it is induced by a lifting function on the vertices of $P$. The set of all lifting functions $\lambda \in \mathbb{R}^{n}$ inducing a fixed regular subdivision $\Sigma_{\lambda}$ is a relatively open polyhedral cone in $\mathbb{R}^{n}$, the secondary cone of $\Sigma_{\lambda}$. The set of all secondary cones forms a polyhedral fan, the secondary fan $\operatorname{SecFan}(P)$. It turns out that the secondary fan is the normal fan of a polytope of dimension $n-d-1$, and any such polytope is a secondary polytope of $P$, that is, the secondary polytope $\operatorname{SecPoly}(P)$ is defined only up to normal equivalence. The vertices of $\operatorname{SecPoly}(P)$ correspond to the regular triangulations of $P$. The reduction in dimension comes from the fact that all the secondary cones in $\operatorname{SecFan}(P)$ have a $(d+1)$-dimensional lineality space in common. By factoring out this lineality space and intersecting with the unit sphere one obtains the spherical polytopal complex $\operatorname{SecFan}^{\prime}(P)$ in $\mathbb{S}^{n-d-2}$. It is dual to the boundary complex of the secondary polytope.

Now fix a Gale dual $G:=\operatorname{Gale}(P)$. Each subset $I \subseteq[n]$ corresponds to a set of (homogenized) vertices $V_{I}$. We set $I^{\star}:=[n] \backslash I$ and $V_{I}^{\star}:=\left\{v_{i}^{\star} \mid i \in I\right\}$. Then the set $V_{I}$ affinely spans $\mathbb{R}^{d}$ if and only if the duals of the complement, that is, the set

$$
V_{I^{\star}}^{\star}=\left\{v_{i}^{\star} \mid i \in[n] \backslash I\right\}
$$

is linearly independent. In particular, for each $d$-dimensional simplex conv $V_{J}$ with $\# J=d+1$ the set pos $V_{J^{\star}}^{\star} \cap \mathbb{S}^{n-d-2}$ is a full-dimensional spherical simplex, which is called the dual simplex of conv $V_{J}$. The chamber complex Chamber $(P)$ is the set of subsets of $\mathbb{S}^{n-d-2}$ arising from the intersections of all the dual simplices. The following theorem by Billera, Gel'fand, and Sturmfels [3] (see also [5, Sect. 5.3]) is essential. 
Theorem 2 ([3, Theorem 3.1]) The chamber complex Chamber $(P)$ is anti-isomorphic to the boundary complex of the secondary polytope $\operatorname{SecPoly}(P)$.

A split of the polytope $P$ is a polytopal decomposition (without new vertices) with exactly two maximal cells. Splits are always regular. The affine hyperplanes weakly separating the two maximal cells of a split are characterized by the property that they do not cut through any edges of $P$ [9, Observation 3.1]; they are called split hyperplanes. Two splits of $P$ are compatible if their split hyperplanes do not intersect in the interior of $P$. They are weakly compatible if they admit a common refinement. Clearly, compatibility implies weak compatibility, but the converse is not true; see Example 3 below. By definition, the splits are coarsest subdivisions of $P$ and hence correspond to rays in the secondary fan or, equivalently, to facets of the secondary polytope and to vertices in the chamber complex. The split complex $\operatorname{Split}(P)$ is the abstract flag-simplicial complex whose vertices are the splits of $P$ which is induced by the compatibility relation. The weak split complex $\operatorname{Split}^{\mathrm{w}}(P)$ is the subcomplex of $\operatorname{SecFan}^{\prime}(P)$ induced by the splits.

Example 3 Let $P=\operatorname{conv}\left\{ \pm e_{i} \mid i \in[d]\right\}$ be a regular crosspolytope in dimension $d$. The splits of $P$ are given by the coordinate hyperplanes $x_{i}=0$, for $i \in[d]$. By combining any $d-1$ of these splits one gets a triangulation of $P$. This shows that the weak split complex is isomorphic to the boundary of a $(d-1)$-simplex. However, any two coordinate hyperplanes contain the origin, whence the corresponding splits are not compatible. The split complex of $P$ has $d$ isolated points. See also [9, Example 4.9].

Proposition 4 The split complex $\operatorname{Split}(P)$ and the weak split complex $\operatorname{Split}^{\mathrm{W}}(P)$ of a polytope $P$ only depend on the oriented matroid of $P$.

Proof Each split $S$ of $P$ defines a cocircuit $C^{\star}$ of the oriented matroid of $P$. A hyperplane which separates $P$ defines a split if and only if it does not separate any edge of $P$. However, an edge of $P$ is a covector of $P$ with exactly two positive entries and it is separated by $S$ if and only if one if the entries is in $C_{+}^{\star}$ and the other is in $C_{-}^{\star}$. So one sees that the set of splits of $P$ only depends on the oriented matroid of $P$.

Now it remains to show that also the compatibility and weak compatibility relations among splits only depend on the oriented matroid.

Let $S_{1}$ and $S_{2}$ be two splits of $P$ with split hyperplanes $H_{S_{1}}$ and $H_{S_{2}}$, respectively. Suppose that $S_{1}$ and $S_{2}$ are incompatible. Then there exists a point $x \in \operatorname{int} P \cap H_{S_{1}} \cap$ $H_{S_{2}}$. Since both split hyperplanes are spanned by vertices of $P$ and since, moreover, each split hyperplane does not intersect any edge, the point $x$ is a convex combination of vertices of $P$ on $H_{S_{1}}$ as well as a convex combination of vertices of $P$ on $H_{S_{2}}$. Thus $x$ gives rise to a vector $C$ in the oriented matroid of $P$ such that $C_{+}$is supported on vertices of $P$ lying on $H_{S_{1}}$ and $C_{-}$is supported on vertices of $P$ lying on $H_{S_{2}}$. That $x$ is contained in the interior of $P$ is equivalent to the property that $C_{+} \cup C_{-}$is not contained in any facet of $P$. Since the facets are precisely the positive cocircuits, it follows that this can be read off from the oriented matroid of $P$.

The statement for the weak split complex follows from the fact that one can construct common refinements of given subdivisions (without new vertices) while only knowing the oriented matroid of the underlying polytope [5, Corollary 4.1.43]. 
Note that, of course, knowing the combinatorics, that is, the face lattice of a polytope is not enough for knowing its split complex or even its splits. As an example consider the regular octahedron (with three splits; see Example 3) and an octahedron with perturbed vertices (which does not have any split). Further, note that the set of regular subdivisions of a polytope does not only depend on the oriented matroid but rather on the coordinatization. So the split subdivisions form a subset of all regular subdivisions which is independent of the coordinatization. In particular, the split complex is a common approximation for the secondary fans of all polytopes with the same oriented matroid but affinely inequivalent coordinates. We continue to use the notation introduced above. In particular, $P$ is the polytope with vertex set $V$ and $G$ a spherical Gale dual.

Remark 5 Whenever there is a circuit $C$ in $G$ with pos $V_{C_{+}}^{\star} \cap \operatorname{pos} V_{C_{-}}^{\star}=\operatorname{pos} x, \operatorname{pos} x$ is a ray in the chamber complex $\operatorname{Chamber}(P)$, arising as the unique intersection ray of the two cells pos $V_{C_{+}}^{\star}$ and pos $V_{C_{-}}^{\star}$. The uniqueness is a consequence of the fact that $C$ is a circuit. Moreover, the subdivision corresponding to the chamber $\{\operatorname{pos} x\}$ contains the corresponding dual cells $\operatorname{conv}\left(V \backslash V_{C_{+}}\right)$and $\operatorname{conv}\left(V \backslash V_{C_{-}}\right)$. Both these cells are full-dimensional because $C$ is a circuit and thus $C_{+}$and $C_{-}$are independent.

The next lemma explains how splits can be recognized in the chamber complex.

Lemma 6 A point $x \in \mathbb{S}^{n-d-2}$ defines a split of $P$ if and only if there exists a unique circuit $C$ in $G$ such that $\operatorname{pos} x=\operatorname{pos} V_{C_{+}}^{\star} \cap \operatorname{pos} V_{C_{-}}^{\star}$.

Proof Consider $x \in \mathbb{S}^{n-d-2}$ such that its chamber is dual to a split $S$ of $P$. Then the split hyperplane $H_{S}$ defines a unique cocircuit $C$ of $P$. Equivalently, $C$ is a circuit of $G$. Moreover, pos $V_{C_{+}}^{\star}$ and pos $V_{C_{-}}^{\star}$ correspond to the two maximal cells of $S$, and $\operatorname{pos} x=\operatorname{pos} V_{C_{+}}^{\star} \cap \operatorname{pos} V_{C_{-}}^{\star}$. Assume there is another circuit $C^{\prime} \neq C$ with pos $V_{C_{+}^{\prime}}^{\star} \cap$ pos $V_{C_{-}^{\prime}}^{\star}=\operatorname{pos} x$. Then, by Remark 5 , the subdivision $S$ of $P$ contains at least four different cells. This is a contradiction since $S$ is split.

Conversely, let $C$ be the unique circuit of $G$ such that $\operatorname{pos} x=\operatorname{pos} V_{C_{+}}^{\star} \cap \operatorname{pos} V_{C_{-}}^{\star}$ for some $x \in \mathbb{S}^{n-d-2}$. By Remark 5, pos $x$ is a ray of the chamber complex, and hence it is dual to a coarsest subdivision $S$ of $P$. By [3, Lemma 3.2], the subdivision $S$ has exactly two maximal cells, since pos $V_{C_{+}}^{\star}$ and pos $V_{C_{-}}^{\star}$ are the only (necessarily minimal) dual cells containing $x$. This means that $S$ is a split.

Example 7 Let $P$ be the pentagon and $G$ its Gale dual from Example 1. Then $C=(0+0--)$ is a cocircuit of $P$ corresponding to the split defined by the line through the vertices $v_{1}$ and $v_{3}$. Clearly, $C$ is also a circuit of $G$, with $C_{+}=\{2\}$ and $C_{-}=\{4,5\}$. We have $\operatorname{pos} v_{2}^{\star}=\operatorname{pos} V_{\{2\}}^{\star} \cap \operatorname{pos} V_{\{4,5\}}^{\star}$, and $C$ is the unique circuit of $G$ yielding pos $v_{2}^{\star}$ as the intersection of its positive and its negative cone. The two maximal cells of the split are the quadrangle conv $V_{\{2\}^{\star}}$ and the triangle conv $V_{\{4,5\}^{\star}}$. See Fig. 1.

With each split $S$ of $P$ we associate the unique circuit $C[S]$ of $G$ from Lemma 6. If $V_{C[S]_{+}}^{\star}$ or $\left(V_{C[S]_{-}}^{\star}\right)$ consists of a single element $v^{\star}$ corresponding to a vertex $v$ of $P$, 
we call $S$ the vertex split for the vertex $v$ and also write $C[v]$ for $C[S]$. Note that the support of $C[v]$ corresponds to the set of all vertices of $P$ that are not connected to $v$ by an edge.

Lemma 8 Let $S$ and $S^{\prime}$ be vertex splits with respect to vertices $v$ and $v^{\prime}$ of $P$. Then $S$ and $S^{\prime}$ are compatible if and only if $v$ and $v^{\prime}$ are not joined by an edge.

Proof It is easily seen that two splits $S, S^{\prime}$ are compatible if and only if (possibly after the negation of one or both of the circuits) $C[S]_{+} \subseteq C\left[S^{\prime}\right]_{+}$and $C\left[S^{\prime}\right]_{-} \subseteq C[S]_{-}$. For a vertex split with respect to the vertex $v$, we have $C[v]_{+}=\left\{v^{\star}\right\}$ or $C[v]_{-}=$ $\left\{v^{\star}\right\}$. However, if $v$ and $v^{\prime}$ are joined by an edge, then $v^{\star} \in C\left[v^{\prime}\right]_{0}$, so the above conditions cannot hold. On the other hand, if $v$ and $v^{\prime}$ are not joined by an edge and, say, $C[v]_{+}=\left\{v^{\star}\right\}$, then (possibly after a negation) $v^{\star} \in C\left[v^{\prime}\right]_{+}$, which implies $\left\{v^{\star}\right\}=C[v]_{+} \subseteq C\left[v^{\prime}\right]_{+}$.

Clearly, $P$ admits a vertex split at the vertex $v$ if and only if the neighbors of $v$ in the vertex-edge graph of $P$ lie on a common hyperplane. In particular, if $P$ is simple then each vertex gives rise to a vertex split.

\section{Totally Splittable Polytopes}

We call a polytope totally splittable if all regular triangulations of $P$ are split triangulations. We aim at the following complete characterization.

Theorem 9 A polytope $P$ is totally splittable if and only if it has the same oriented matroid as a simplex, a crosspolytope, a polygon, a prism over a simplex, or a (possibly multiple) join of these polytopes.

By Proposition 4, the set of splits and their (weak) compatibility only depends on the oriented matroid of $P$, and hence the notion totally splittable also depends on the oriented matroid only. The join $P * Q$ of a $d$-polytope $P$ and an $e$-polytope $Q$ is the convex hull of $P \cup Q$, seen as subpolytopes in mutually skew affine subspaces of $\mathbb{R}^{d+e+1}$. For instance, a 3-simplex is the join of any pair of its disjoint edges. In order to avoid cumbersome notation in the remainder of this section, we do not distinguish between any two polytopes sharing the same oriented matroid. For instance, " $P$ is a join of $P_{1}$ and $P_{2}$ " actually means " $P$ has the same oriented matroid as the join of $P_{1}$ and $P_{2} "$ and so on.

Example 10 We inspect the classes of polytopes occurring in Theorem 9.

(i) Simplices are totally splittable in a trivial way.

(ii) A triangulation of an $n$-gon is equivalent to choosing $n-3$ diagonals which are pairwise non-intersecting. This is a compatible system of splits, and hence each polygon is totally splittable; see [9, Example 4.8]. The secondary polytope of an $n$-gon is the $(n-3)$-dimensional associahedron [6, Chapter 7, Sect. 3.B]. 

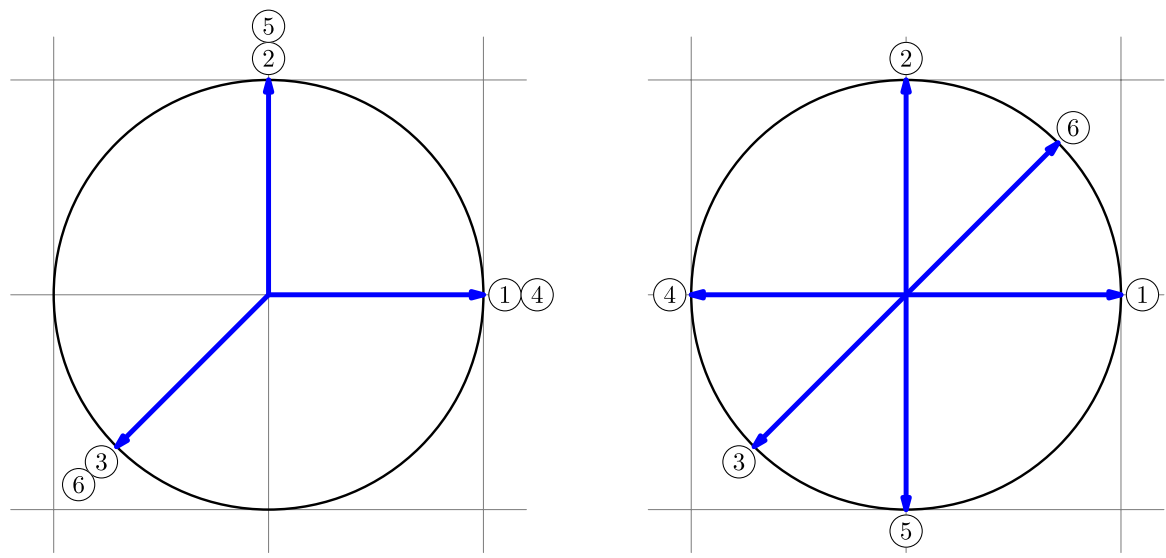

Fig. 2 Gale diagrams of the regular octahedron (left) and of the prism over a triangle (right)

(iii) Let $P=\operatorname{conv}\left\{ \pm e_{i} \mid i \in[d]\right\}$ be a regular crosspolytope in dimension $d$ as in Example 3 . The splits correspond to the coordinate hyperplanes, and any $d-1$ of them induce a triangulation of $P$. Conversely, each triangulation of $P$ arises in this way. See [9, Example 4.9]. A Gale dual of $P$ is given by the multiset $G \subset \mathbb{S}^{d-2}$ consisting of all points

$$
\left\{e_{i} \mid i \in[d-1]\right\} \cup\left\{-\frac{1}{\sqrt{d-1}} \sum_{i=1}^{d-1} e_{i}\right\},
$$

where each point occurs exactly twice. All the vertices in the chamber complex correspond to vertex splits, and the chamber complex is the normal fan of a $(d-1)$-simplex (where each vertex carries two labels). So the secondary polytope of $P$ is a $(d-1)$-simplex. See Fig. 2 (left) below for $d=3$.

(iv) Let $P$ be the prism over a $(d-1)$-simplex. Then the dual graph of any triangulation of $P$ is a path with $d$ nodes. The secondary polytope of $P$ is the $(d-1)$-dimensional permutohedron [6, Chapter 7, Sect. 3.C]. See Fig. 2 (right) above for $d=3$.

Remark 11 As the secondary polytope of a join of polytopes is the product of their secondary polytopes (e.g., this can be inferred from [5, Corollary 4.2.8]), Theorem 9 and Example 10 show that the secondary polytopes of totally splittable polytopes are (possibly multiple) products of simplices, permutohedra, and associahedra.

Remark 12 One can ask the question: What is the typical behavior of a polytope in terms of splits? The smallest example of a polytope that does not have any split is given by an octahedron whose vertices are slightly perturbed into general position. Moreover, any 2-neighborly polytope (that is, any two vertices share an edge) does not admit any split [9, Proposition 3.4]. On the other hand, $d$-dimensional simple polytopes with $n$ vertices have at least $n$ splits: Each vertex is connected to exactly $d$ other vertices which span a split hyperplane for the corresponding vertex split. This shows that the answer of the seemingly more precise question of how many splits 
is a "random polytope" expected to have highly depends on the chosen model. On the one hand, a $d$-polytope whose facets are chosen uniformly at random tangent to the unit sphere is simple with probability one; hence it has at least as many splits as vertices. On the other hand, one can choose models such that the polytopes generated are 2-neighborly with high probability [11]; such polytopes do not have any splits.

It is obvious that total splittability is a severe restriction among polytopes. The following result is a key first step. As an essential tool we use that any ordering of the vertices of a polytope induces a triangulation, the placing triangulation with respect to that ordering [5, Sect. 4.3.1]. Moreover, successive placing of new vertices can be used to extend any triangulation of a subpolytope.

Proposition 13 Let $P$ be a totally splittable polytope. Then each face, each vertex figure, and each subpolytope $Q:=\operatorname{conv}(V \backslash\{v\})$ for a vertex $v \in V$ is totally splittable. Moreover, $v$ gives rise to a vertex split, and the neighbors of $v$ span a facet of $Q$.

Proof Let $\Delta$ be an arbitrary triangulation of a facet $F$ of $P$. We have to show that $\Delta$ is induced by splits of $F$. By placing the vertices of $P$ not in $F$ in an arbitrary order we can extend $\Delta$ to a triangulation $\Delta^{\prime}$ of $P$. As $P$ is totally splittable $\Delta^{\prime}$ is induced by splits of $P$. A split of $P$ either does not separate $F$, or it is a split of $F$. This implies that $\Delta$ is induced by splits of $F$. Inductively, this shows the total splittability of all faces of $P$.

Consider the subpolytope $Q:=\operatorname{conv}(V \backslash\{v\})$ for some vertex $v$ of $P$. We can assume that $P$ is not a simplex, hence $Q$ is full-dimensional. Take an arbitrary triangulation $\Sigma$ of $Q$. By placing $v$ this extends to a triangulation $\Sigma^{\prime}$ of $P$. The $d$-simplices of $\Sigma^{\prime}$ containing $v$ are the cones (with apex $v$ ) over those codimension $(d-1)$ )-faces of $\Sigma$ which span a hyperplane weakly separating $Q$ from $v$. By assumption, $\Sigma^{\prime}$ is a split triangulation, and hence each interior cell of codimension one spans a split hyperplane. Fix a $d$-simplex $\sigma \in \Sigma^{\prime}$ containing $v$. The facet of $\sigma$ not containing $v$ is an interior cell of codimension one, which is why it spans a split hyperplane $H$. Since $H$ cannot cut through the other simplices in $\Sigma^{\prime}$ all neighbors of $v$ in the vertex-edge graph of $P$ are contained in $H$. This proves that $H$ is the split hyperplane of the vertex split to $v$, and $H$ intersects $Q$ in a facet. This also shows that the triangulation $\Sigma$ of $Q$ is induced by splits of $Q$, and $Q$ is totally splittable.

The vertex figure of $P$ at $v$ is affinely equivalent to the facet $Q \cap H$ of $Q$, and hence the total splittability of the vertex figure follows from the above.

Remark 14 The same argument as in the proof above shows: Each hyperplane spanned by $d$ affinely independent vertices of a totally splittable polytope defines a facet or a split.

Note that there exist polytopes for which each vertex defines a vertex split, but which are not totally splittable. An example is the 3-cube which is simple, and hence each vertex defines a vertex split [9, Remark 3.3], but which has several triangulations which are not induced by splits [9, Examples 3.8 and 4.10]. It is crucial that, by 
Proposition 13, the neighbors of a vertex $v$ of a totally splittable polytope span a hyperplane, which we denote by $v^{\perp}$. Two vertices of $P$ are neighbors if they share an edge $w$ in the vertex-edge graph of $P$. Proposition 13 makes it possible to re-read Lemma 6 as follows.

Corollary 15 Let $v$ be a vertex of a totally splittable polytope P. Then

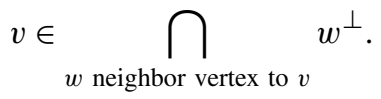

Remark 16 In the situation of Proposition 13, all facets of $Q$ are also facets of $P$ except for the facet $F$ spanning the hyperplane $v^{\perp}$. Moreover, all vertices of $Q$ are also vertices of $P$. In this situation, we say that $v$ is almost beyond the facet $F$ of $Q$. This is slightly more general than requiring $v$ to be beyond $Q$, which means that $F$ is the unique facet of $Q$ violated by $v$, and additionally $v$ is not contained in any hyperplane spanned by a facet of $Q$. That $F$ is violated by $v$ means that the closed affine halfspace with boundary hyperplane aff $F$ does not contain the point $v$. If $v$ is beyond $F$ and $d=\operatorname{dim} P=\operatorname{dim} Q \geq 3$ then the vertex-edge graph of $Q$ is the subgraph of the vertex-edge graph of $P$ induced on Vert $P \backslash\{v\}=\operatorname{Vert} Q$. The neighbors of $v$ are precisely the vertices on the facet $F$ of $Q$.

Lemma 17 For two polytopes $P$ and $Q$ the join $P * Q$ is totally splittable if and only if both $P$ and $Q$ are.

Proof Suppose that $P * Q$ is totally splittable. Then $P$ and $Q$ both occur as faces of $P * Q$, and the claim follows from Proposition 13 .

Let $\operatorname{dim} P=d$ and $\operatorname{dim} Q=e$, and assume that $P$ and $Q$ both are totally splittable. The join of a $d$-simplex and an $e$-simplex is a $(d+e+1)$-simplex, and hence the join cell-by-cell of a triangulation of $P$ and a triangulation of $Q$ yields a triangulation of $P * Q$. Conversely, each triangulation of $P * Q$ arises in this way [5, Theorem 4.2.7]. The join of a split hyperplane of $P$ with aff $Q$ and the join of a split hyperplane of $Q$ with aff $P$ yields split hyperplanes of $P * Q$. Now consider any triangulation $\Delta$ of $P * Q$. Then there are triangulations $\Delta_{P}$ and $\Delta_{Q}$ of $P$ and $Q$, respectively, such that $\Delta=\Delta_{P} * \Delta_{Q}$. By assumption, there is a set $S_{P}$ of splits of $P$ inducing $\Delta_{P}$. Likewise, $S_{Q}$ is the set of splits inducing $\Delta_{Q}$. Then the set of joins of all splits from $S_{P}$ with aff $Q$ (as an affine subspace of $\mathbb{R}^{d+e+1}$ ) and the set of joins of all splits from $S_{Q}$ with aff $P$ jointly induce the triangulation $\Delta$.

Lemma 17 together with Example 10 completes the proof that all the polytopes listed in Theorem 9 are, in fact, totally splittable. The remainder of this section is devoted to proving that there are no others.

Proposition 18 Let $P \subset \mathbb{R}^{d}$ be a proper totally splittable $d$-polytope. Then $P$ is a regular crosspolytope if and only if the intersection $\bigcap_{v \in \operatorname{Vert} P} v^{\perp}$ is not empty.

Proof Clearly, the regular crosspolytope $P=\operatorname{conv}\left\{ \pm e_{i} \mid i \in[d]\right\}$ has the property that the intersection of its split hyperplanes is the origin. Conversely, suppose that $P$ 
is not a crosspolytope. We assumed that $P$ is proper, meaning that $P$ is not a pyramid. Hence there exists a vertex $v$ of $P$ such that at least two vertices $u, w$ are separated from $v$ by the hyperplane $v^{\perp}$. By Proposition 13, the split hyperplane $v^{\perp}$ passes through the neighbors of $v$ in the vertex-edge graph of $P$. Since $u$ is on the same side of $v^{\perp}$ as $w$ it follows that $v^{\perp} \neq w^{\perp}$ and, moreover, $v^{\perp} \cap w^{\perp} \cap$ int $P=\emptyset$. Now suppose that the intersection of all split hyperplanes contains points in the boundary of $P$. But since the split hyperplanes do not cut through edges, the intersection must contain at least one vertex $x \in \operatorname{Vert} P$. This is a contradiction since $x \notin x^{\perp}$. By a similar argument, we can exclude the final possibility that the intersection of all split hyperplanes contains any points outside $P$. Therefore, this intersection is empty, as we wanted to show.

In a way, crosspolytopes (which are not quadrangles) are maximally totally splittable.

Lemma 19 Let $P \subset \mathbb{R}^{d}$ be a d-dimensional regular crosspolytope and $v \in \mathbb{R}^{d} \backslash P$ be a point almost beyond the facet $F$ of $P$. If $d \geq 3$ then $\operatorname{conv}(P \cup\{v\})$ is not totally splittable.

Proof Without loss of generality, $P=\operatorname{conv}\left\{ \pm e_{1}, \pm e_{2}, \ldots, \pm e_{d}\right\}$. Suppose that $\operatorname{conv}(P \cup\{v\})$ is totally splittable. Since we assumed $d \geq 3$, each vertex $w$ of $P$ has at least $d+1$ neighbors. At least $d$ affinely independent vertices among these are still neighbors of $w$ in $\operatorname{conv}(P \cup\{v\})$, so the hyperplane $w^{\perp}$ with respect to $P$ is the same as $w^{\perp}$ with respect to $\operatorname{conv}(P \cup\{v\})$. We have that $F^{\perp}:=\bigcap_{w \in \operatorname{Vert}} w^{\perp}=\{0\}$, which implies $v \notin F^{\perp}$, a contradiction to Corollary 15 .

The same conclusion as in Lemma 19 holds for prisms over simplices as well. See also Fig. 3 and Example 21 below.
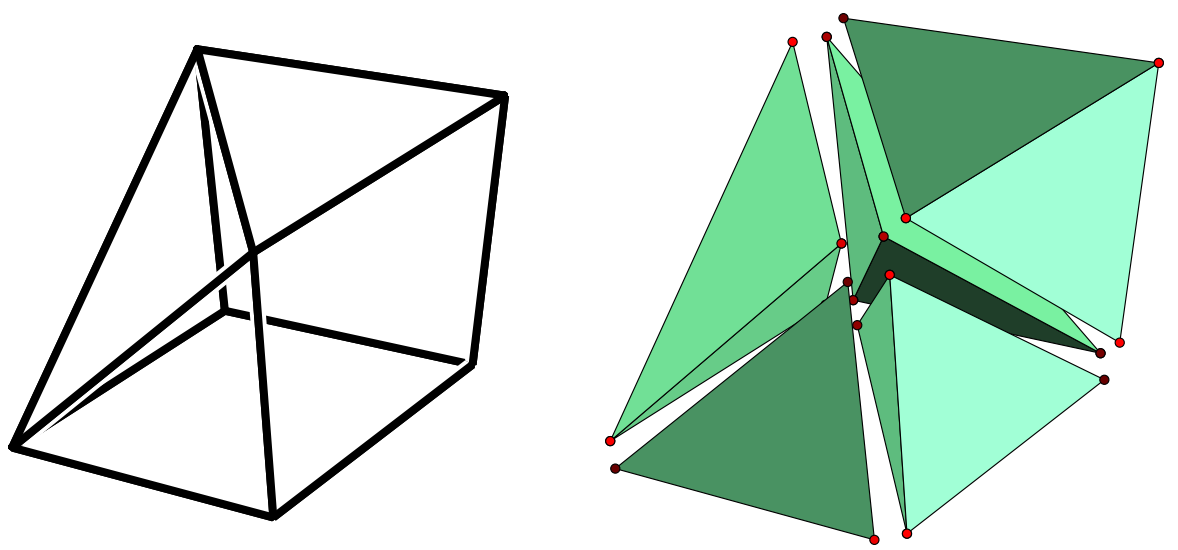

Fig. 3 Convex hull of prism plus one point almost beyond a quadrangular facet, vertex-edge graph (left) and a non-split triangulation (right) 
Lemma 20 Let $P \subset \mathbb{R}^{d}$ be a prism over a $(d-1)$-simplex and $v \in \mathbb{R}^{d} \backslash P$ a point which is almost beyond a facet $F$ of $P$. If $d \geq 3$ then $\operatorname{conv}(P \cup\{v\})$ is not totally splittable.

Proof Suppose that $\operatorname{conv}(P \cup\{v\})$ is totally splittable. As in the proof of Lemma 19 we are aiming at a contradiction to Corollary 15. First, suppose that $v$ is beyond $F$, and hence for $w \in$ Vert $P$ the hyperplanes $w^{\perp}$ with respect to $P$ and $\operatorname{conv}(P \cup\{v\})$ coincide, since $d \geq 3$; see Remark 16. Up to an affine transformation, we can assume that $P=\operatorname{conv}\left\{e_{1}, e_{2}, \ldots, e_{d}, f_{1}, f_{2}, \ldots, f_{d}\right\}$ with

$$
f_{k}=-\sum_{i \neq k} e_{i}
$$

The neighbors of the vertex $e_{k}$ are $e_{1}, e_{2}, \ldots, e_{k-1}, e_{k+1}, \ldots, e_{d}$, and $f_{k}$; symmetrically for the $f_{k}$. A direct computation shows that

$$
e_{k}^{\perp}=\left\{x \mid x_{k}=0\right\} \quad \text { and } \quad f_{k}^{\perp}=\left\{x \mid 2 \sum_{i \neq k} x_{i}=(d-2)\left(x_{k}-1\right)\right\} .
$$

We have to distinguish two cases: The facet $F$ of $P$ violated by $v$ may be a $(d-1)$-simplex or a prism over a $(d-2)$-simplex. If $F$ is a simplex, for instance, $\operatorname{conv}\left\{e_{1}, e_{2}, \ldots, e_{d}\right\}$, then we can conclude that the set $\bigcap_{w \in F} w^{\perp}=\{0\}$ which is in the interior of $P$ and hence cannot be equal to $v$. If, however, $F$ is a prism, for instance, with the vertices $e_{1}, e_{2}, \ldots, e_{d-1}, f_{1}, f_{2}, \ldots, f_{d-1}$, we can compute that

$$
\bigcap_{w \in \operatorname{Vert} F} w^{\perp}=\left\{\frac{2-d}{2} e_{d}\right\}
$$

again an interior point. In both cases, we arrive at the desired contradiction to Corollary 15.

Now suppose that $v$ violates $F$ but it is not beyond $F$, that is, $v$ is contained in the affine hull of some facet $F^{\prime}$ of $P$. Let us assume that $d \geq 4$ and that the assertion is true for $d=3$. Then the polytope $\operatorname{conv}\left(F^{\prime} \cup\{v\}\right)$ is totally splittable by Proposition 13. Again, $F^{\prime}$ may be a $(d-1)$-simplex or a prism over a $(d-2)$-simplex. If $F^{\prime}$ is a $(d-1)$-simplex, it can easily be seen that $\operatorname{conv}\left(F^{\prime} \cup\{v\}\right)$ is not totally splittable for $d>3$ since $F^{\prime}$ does not have any splits. If $F^{\prime}$ is a prism over a simplex, we are done by induction.

An easy consideration of the cases, which we omit, allows us to prove the result in the base case $d=3$. See Example 21 and Fig. 3 for one of the cases arising.

Example 21 Consider the 3-polytope $P=\operatorname{conv}\left\{e_{1}, e_{2}, e_{3},-e_{2}-e_{3},-e_{1}-e_{3},-e_{1}-e_{2}\right\}$, which is a prism over a triangle. For instance, the point $v=e_{1}+e_{2}-e_{3}$ lies almost beyond the quadrangular facet $F=\operatorname{conv}\left\{e_{1}, e_{2},-e_{2}-e_{3},-e_{1}-e_{3}\right\}$. The polytope $\operatorname{conv}(P \cup\{v\})$ admits a triangulation which is not split; see Fig. 3.

Proposition 22 Let $P$ be a proper totally splittable polytope that is not a regular crosspolytope. Then $P$ is a join if and only if the vertex set of $P$ admits a partition 
Vert $P=U \cup W$ such that no vertex split of a vertex in $U$ is compatible with any vertex split of a vertex in $W$.

Proof Let $P=(\operatorname{conv} U) *(\operatorname{conv} W)$ be a proper join. In particular, $P$ is not a pyramid, and conv $U$ and conv $W$ both are at least one-dimensional. Then, by the definition of join, each vertex in $U$ shares an edge with each vertex in $W$, and thus the corresponding vertex splits are not compatible.

Conversely, assume that no split with respect to a vertex in $U$ is compatible with a split with respect to any vertex in $W$. By Lemma 8, each vertex in $U$ is joined by an edge to each vertex in $W$. Proposition 13 says that each vertex split hyperplane $u^{\perp}$ contains all neighbors of $u$. Thus we infer that $\bigcap_{u \in U} u^{\perp} \supset$ conv $W$ and, symmetrically, $\bigcap_{w \in W} w^{\perp} \supset \operatorname{conv} U$. Now there are two cases to distinguish. If $\bigcap_{v \in \operatorname{Vert} P} v^{\perp}$ is non-empty then $P$ is a regular crosspolytope due to Proposition 18, contradicting the assumption. The remaining possibility is that $\bigcap_{v \in \operatorname{Vert} P} v^{\perp}$ is empty. In this case we have

$$
\text { aff } U \cap \operatorname{aff} W \subseteq \bigcap_{w \in W} w^{\perp} \cap \bigcap_{u \in U} u^{\perp}=\bigcap_{v \in \operatorname{Vert} P} v^{\perp}=\emptyset .
$$

The affine subspaces aff $U$ and aff $W$ are skew. It follows that $P=(\operatorname{conv} U) *$ $(\operatorname{conv} W)$.

For the following, we will switch from the primal view on our polytope $P$ to its spherical Gale dual $G$. A point of multiplicity two in $G$ is called a double point. Vertices of $P$ corresponding to the same point in $G$ are called siblings.

Lemma 23 Let $P$ be a totally splittable polytope which is not a join, and let $G$ be a spherical Gale diagram of $P$. Then $P$ is proper, and each point of $G$ is a single point, or each point is a double point. In particular, there are no points in $G$ with multiplicity greater than two.

Proof If $P$ is a regular crosspolytope, we know from the explicit description of $G$ in Example 10(iii) that the conclusion of the lemma holds. So we can assume that this is not the case. Since we assume that $P$ is not a join, in particular, it is not a pyramid, and this is why $P$ is proper. If $G$ has a point with multiplicity three or above, then each pair of copies of $x$ defines a circuit, which yields a contradiction to Lemma 6 .

So suppose now that $v_{1}$ is a vertex that has a sibling $v_{2}$ and that the set $W$ of all vertices without a sibling is non-empty. Then, again by Lemma $6, v_{1}^{\star}=v_{2}^{\star}$ is not contained in pos $W^{\star}$. By the Separation Theorem [7, 2.2.2], there is an affine hyperplane in $\mathbb{R}^{n-d-1}$ which weakly separates $v_{1}^{\star}=v_{2}^{\star}$ from pos $W^{\star}$. This argument even works for all vertices with a sibling simultaneously. That is, $H$ weakly separates the double points from non-double points. By rotating $H$ slightly, if necessary, we can further assume that $H$ contains at least one dual vertex $w^{\star}$ of a vertex $w \in W$ without a sibling. For each such $w \in W$ with $w^{\star} \in H$, the support of the circuit $C[w]$ is a subset of $W^{\star}$, and from Lemma 6 it follows that the support of $C[w]$ is contained in the hyperplane $H$. In the primal view, this means that all vertices $v$ of $P$ with $v^{\star} \notin H$ have to be in the splitting hyperplane $w^{\perp}$ and that the vertex split of $w$ 
Fig. 4 Gale diagram of the join of two squares, labeled $\{1,2,3,4\}$ and $\{5,6,7,8\}$, respectively

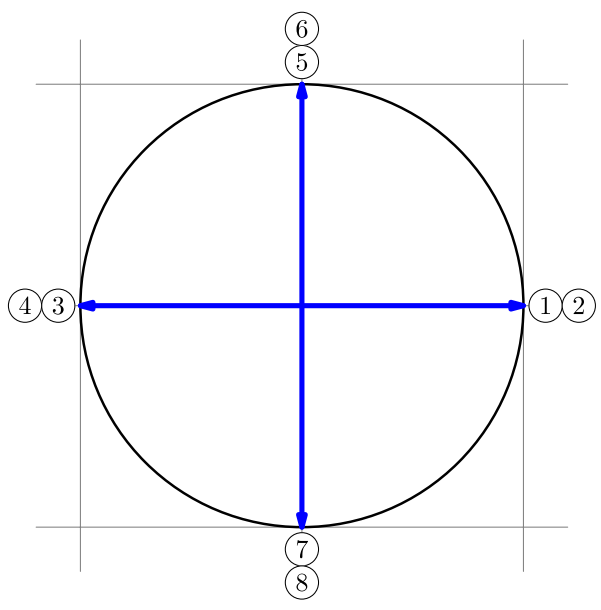

cannot be compatible to any vertex split of a vertex $v$ with $v^{\star} \notin H$. If now we define $U:=\left\{w \in \operatorname{Vert} P \mid w^{\star} \in H\right\}$, we have a partition of Vert $P$ in $U$ and Vert $P \backslash U$ such that no vertex split of a vertex in $U$ is compatible with any vertex split of a vertex in Vert $P \backslash U$. So $P$ is a join by Proposition 22 .

A point $x \in G$ is antipodal if $-x$ is also in $G$. Notice that each quadrangle, regular or not, has a zero-dimensional spherical Gale diagram with exactly two pairs of antipodal points. Figure 4 shows a Gale diagram of the join of two squares.

Lemma 24 Let $P$ be a totally splittable $d$-polytope with $d \geq 2$ which is not a join. If each point in the spherical Gale diagram $G$ is a double point then $P$ is a regular crosspolytope.

Proof Assume that each point in $G$ is a double point. Let $v$ be any vertex of $P$ and $v^{\perp}$ the hyperplane corresponding to the vertex split of $v$. Since $v^{\star}$ is a double point in $G$, there is exactly one vertex $w$ other than $v$ which is not contained in $v^{\perp}$. The polytope $Q:=\operatorname{conv}($ Vert $P \backslash\{v, w\})=P \cap v^{\perp}$ is a face of the vertex figure of $v$, and hence totally splittable by Proposition 13. Clearly, a spherical Gale diagram of $Q$ again has only double points. Inductively, we can thus assume that $Q$ is a regular crosspolytope. Therefore, its split hyperplanes have a non-empty intersection. Since this intersection is contained in $v^{\perp}$, it follows that the split hyperplanes of $P$ also have a non-empty intersection. Hence $P$ is a regular crosspolytope by Proposition 18. As a basis of the induction we can consider the case where $G$ is contained in $\mathbb{S}^{1}$. As $G$ must span $\mathbb{R}^{2}$, and as each point in $G$ occurs twice, the polytope $P$ has six vertices, and it is threedimensional. It can be shown that $P$ is a regular octahedron. The two-dimensional case will be dealt with in the proof of Lemma 26 below.

Lemma 25 Let $P$ be a totally splittable $d$-polytope with $d \geq 2$ which is not a join. If each point in the spherical Gale diagram $G$ is antipodal then $P$ is a prism over a simplex. 
Proof Suppose that each point in $G$ is antipodal. Let $k:=n-d-1$ be the dimension of the linear span of $G$, and so we can view $G$ as a subset of $\mathbb{S}^{k-1}$. We claim that the number of vertices of $P$ equals $n=2 d$ or, equivalently, that $n=2 k+2$. Pick any cocircuit of $G$. This corresponds to a linear hyperplane $H$ in $\mathbb{R}^{k}$ which contains at least $2 k-2$ points of $G$, due to antipodality. Since $G$ is the Gale diagram of a polytope, each open halfspace defined by $H$ contains at least 2 points [12, Theorem 6.19]. We conclude that $n \geq 2 k+2$.

Now we will show that $n \leq 2 k+2$, hence $n=2 k+2$. To arrive at a contradiction, suppose that the spherical Gale diagram $G$ contains at least $k+2$ antipodal pairs. Take any vertex $v$ of $P$, and let $v^{\star}$ be its dual in $G$. Let $v_{1}, v_{2}, \ldots, v_{r}$ be those vertices of $P$ which are not joined to $v$ by edges. Then the circuit $C[v]$ of $G$ corresponding to the vertex split of $v$ has the support $\left\{v^{\star}, v_{1}^{\star}, v_{2}^{\star}, \ldots, v_{r}^{\star}\right\}$. In particular, $v^{\star}$ is contained in the positive hull of $v_{1}^{\star}, v_{2}^{\star}, \ldots, v_{r}^{\star}$. Pick a linear hyperplane in $\mathbb{R}^{k}$ in general position with respect to the points of $G$ which has the cone $\operatorname{pos}\left\{v_{1}^{\star}, v_{2}^{\star}, \ldots, v_{r}^{\star}\right\}$ on one side. Let $H^{\star}$ be a parallel affine hyperplane such that $v^{\star}$ and the origin are on different sides of $H^{\star}$. Let $W=\left\{w_{1}^{\star}, w_{2}^{\star}, \ldots, w_{m}^{\star}\right\}$ be the set of points in $G$ distinct from $v^{\star}$ for which the corresponding rays intersect $H^{\star}$. Firstly, $m \geq k+1$ since $G$ contains $k+1$ antipodal pairs in addition to $v^{\star}$ and its antipode. Secondly, $v^{\star}$ is in the positive span pos $W$ of the points in $W$ since among those points are the points $v_{1}^{\star}, v_{2}^{\star}, \ldots, v_{r}^{\star}$. Thirdly, the cone pos $W$ is $k$-dimensional since $G$ positively spans $\mathbb{R}^{k}$ and $W$ contains exactly one point from each antipodal pair in $G$. By Carathéodory's Theorem [7, Sect. 2.3.5] we can assume that the cone spanned by $W^{\prime}:=\left\{w_{1}^{\star}, w_{2}^{\star}, \ldots, w_{k+1}^{\star}\right\}$ still contains $v^{\star}$. Let $Q$ be the intersection of pos $W^{\prime}$ with the hyperplane $H^{\star}$. By construction, $Q$ is a $(k-1)$-dimensional polytope with $k+1$ vertices. Such a polytope has precisely two triangulations $\Delta$ and $\Delta^{\prime}$; these are related by a flip, see [5, Sect. 2.4.1]. Let $\sigma$ and $\sigma^{\prime}$ be maximal simplices of $\Delta$ and $\Delta^{\prime}$ containing the point $\left(\mathbb{R} v^{\star}\right) \cap H^{\star}$. By construction, $\sigma$ gives rise to a circuit $D$ of $G$ whose negative support corresponds to the vertices of $\sigma$, and its positive support corresponds to $v^{\star}$. Similarly, $\sigma^{\prime}$ defines another such circuit $D^{\prime}$. Since no maximal simplex of $\Delta$ also occurs as a maximal simplex in $\Delta^{\prime}$, we have $\sigma \neq \sigma^{\prime}$, implying $D \neq D^{\prime}$. This contradicts Lemma 6 , and this finally proves that $n$ equals $2 k+2$.

By now we know that $G$ consists of precisely $k+1$ antipodal pairs in $\mathbb{S}^{k-1}$. So $P$ is a $d$-polytope with $2 k+2=2 d$ vertices. We have to show that $P$ has the same oriented matroid as a prism over a $(d-1)$-simplex. This will be done by showing that the cocircuits of $G$ (which are the circuits of $P$ ) agree with the circuits of a prism over a simplex. So consider the prism over a simplex with coordinates as in the proof of Lemma 20. Then each circuit $C$ of this prism is of the form

$$
C_{+}=\left\{e_{i}, f_{j}\right\} \quad \text { and } \quad C_{-}=\left\{f_{i}, e_{j}\right\}
$$

for distinct $i$ and $j$. Moreover, $e_{i}^{\star}$ and $f_{i}^{\star}$ are antipodes in the prism's spherical Gale diagram. The cocircuits of $G$ are given by all (linear) hyperplanes in $\mathbb{R}^{k}$ spanned by $k-1$ pairs of points in $G$. None of the other two pairs of points can be contained in such a hyperplane since $G$ is the Gale diagram of a polytope [12, Theorem 6.19]. So the cocircuits of $G$ are given by $C_{+}^{\star}=\{x, y\}, C_{-}^{\star}=\{-x,-y\}$ for all distinct $x, y \in G$ with $x \neq \pm y$. 
Lemma 26 Let $P$ be a totally splittable $d$-polytope with $d \geq 2$ which is not a join. If each point in the spherical Gale diagram $G$ is both a double point and antipodal then $d=2$, and $P$ is a quadrangle.

Proof If each point in $G$ is antipodal from Lemma 25, we know that $P$ is a prism over a $(d-1)$-simplex. The only case in which such a Gale diagram has the property that each point is a double point is $d=2$, and $P$ is a quadrangle.

Now we have all ingredients to prove our main result.

Proof of Theorem 9 Let $P$ be a totally splittable $d$-polytope with spherical Gale dual $G$. By Lemma 17, we can assume without loss of generality that $P$ is not a join. Consider a vertex $v \in \operatorname{Vert} P$ with the property that $v^{\star}$ is neither a double nor an antipodal point. By Proposition 13, the polytope $Q:=\operatorname{conv}($ Vert $P \backslash\{v\}$ ) obtained from $P$ by the deletion of $v$ is again totally splittable. Moreover, $\operatorname{dim} Q=d$ since $P$ is not a pyramid.

Let us assume for the moment that $Q$ is also not a join. Then we can repeat this procedure until after finitely many steps we arrive at a polytope $P^{\prime}$ with a spherical Gale diagram $G^{\prime}$ which consists only of double and antipodal points. In this situation, Lemma 23 implies that all points of $G^{\prime}$ are double points or all points of $G^{\prime}$ are antipodal. Combining Lemmas 24, 25, and 26, we can conclude that either $d=\operatorname{dim} P=\operatorname{dim} P^{\prime}=2$ and $P^{\prime}$ is a quadrangle, or $d \geq 3$ and $P^{\prime}$ is a regular crosspolytope, or $d \geq 3$ and $P^{\prime}$ is a prism over a simplex. The question remaining is whether $P$ and $P^{\prime}$ can actually be different. For $d \geq 3$ this is ruled out by Lemma 19 (if $P^{\prime}$ is a crosspolytope) and Lemma 20 (if $P^{\prime}$ is a prism). In the final case, $\operatorname{dim} P=\operatorname{dim} Q=\operatorname{dim} P^{\prime}=2$.

The proof of our main result will be concluded with the subsequent proposition.

Proposition 27 Let $P$ be a totally splittable polytope with spherical Gale diagram $G$, and let $v$ be a vertex of $P$ with the property that its dual $v^{\star}$ in $G$ is neither a double nor an antipodal point. If $P$ is not a join then neither is $Q:=\operatorname{conv}($ Vert $P \backslash\{v\}$ ).

Proof By [3, Lemma 3.4], the Gale transform of $Q$ is the minor $G / v^{\star}$ obtained by contracting $v^{\star}$ in $G$. Up to an affine transformation, we can assume that $v^{\star}$ is the first unit vector in $\mathbb{R}^{n-d-1}$, and so $G / v^{\star}$ is the projection of $G \backslash\left\{v^{\star}\right\}$ to the last $n-d-2$ coordinates. We call the projection map $\pi$. Since $v^{\star}$ is neither antipodal nor a double point, no point in $G / v^{\star}$ is a loop, and thus $Q$ is proper, that is, it is not a pyramid.

So suppose that $Q=Q_{1} * Q_{2}$ is a join with $\operatorname{dim} Q_{1} \geq 1$ and $\operatorname{dim} Q_{2} \geq 1$. Then there are spherical Gale diagrams $G_{1}$ and $G_{2}$ of $Q_{1}$ and $Q_{2}$, respectively, such that $G / v^{\star}=G_{1} \sqcup G_{2}$ as a multiset in $\mathbb{S}^{n-d-3}$. Up to exchanging the roles of $Q_{1}$ and $Q_{2}$, there is a facet $F_{1}$ of $Q_{1}$ such that $v^{\perp} \cap P$, which is a facet of $Q$, is a join $F_{1} * Q_{2}$. That is to say, the cosupport of the circuit $C[v]$, corresponding to the vertex split of $v$ in $P$, is mapped to $G_{1}$ by $\pi$. In particular, $v^{\star}$ is not in the positive hull of the points dual to the vertices of $Q_{2}$. The Separation Theorem [7, 2.2.2] implies that there is a linear hyperplane $H$ in $\mathbb{R}^{n-d-1}$ separating $v^{\star}$ from the duals of the vertices of $Q_{2}$. 
As in the proof of Lemma 23 we can now argue that $P$ is a join, which contradicts our assumptions.

This finally completes the proof of the theorem.

Remark 28 If $v^{\star}$ is antipodal or a double point, then $Q$ is a pyramid over the unique facet of $Q$ which is not a facet of $P$. This shows that the assumption on $v^{\star}$ in Proposition 27 is necessary. For instance, by inspecting the two Gale diagrams in Fig. 2 one can see directly that if $P$ is a regular octahedron or a prism over a triangle, in both cases $Q$ is a pyramid over a quadrangle.

Remark 29 A triangulation $\Delta$ of a $d$-polytope is foldable if the dual graph of $\Delta$ is bipartite. This is equivalent to the property that the 1-skeleton of $\Delta$ is $(d+1)$ colorable. In [9, Corollary 4.12], it was proved that any triangulation generated by splits is foldable. This means that each triangulation of a totally splittable polytope is necessarily foldable.

We are indebted to Raman Sanyal for sharing the following observation with us.

\section{Corollary 30 Each totally splittable polytope is equidecomposable.}

A polytope is equidecomposable if each triangulation has the same $f$-vector.

Proof This follows from the classification case by case: Each triangulation of an $n$-gon has exactly $n-2$ triangles. Each triangulation of a $d$-dimensional regular crosspolytope has exactly $2 d-2$ maximal cells. Each triangulation of a prism over a $(d-1)$-simplex has exactly $d$ maximal cells. A similar count can be done for the lower dimensional cells. Observe that equidecomposability is preserved under taking joins.

It would be interesting to know if Corollary 30 has a direct proof without relying on Theorem 9.

Remark 31 Bayer [2] defines a polytope to be weakly neighborly if any $k$ of its vertices are contained in some face of dimension $2 k-1$. She shows that a weakly neighborly polytope is necessarily equidecomposable [2, Corollary 10]. Prisms over simplices are weakly neighborly whereas crosspolytopes are not; so the approach of Bayer is somewhat transverse to ours. Moreover, all circuits of a totally splittable polytope are balanced in the sense that the positive and the negative support share the same cardinality. This relates to the question of whether a polytope all of whose circuits are balanced is always equidecomposable. The converse is true [2, Theorem 1].

\section{References}

1. Bandelt, H.-J., Dress, A.: A canonical decomposition theory for metrics on a finite set. Adv. Math. 92(1), 47-105 (1992). MR 1153934 (93h:54022)

2. Bayer, M.M.: Equidecomposable and weakly neighborly polytopes. Israel J. Math. 81(3), 301-320 (1993). MR 1231196 (94m:52015) 
3. Billera, L.J., Gel'fand, I.M., Sturmfels, B.: Duality and minors of secondary polyhedra. J. Combin. Theory, Ser. B 57(2), 258-268 (1993). MR 1207491 (93m:52014)

4. Björner, A., Las Vergnas, M., Sturmfels, B., White, N., Ziegler, G.M.: Oriented Matroids, 2nd edn. Encyclopedia of Mathematics and its Applications, vol. 46. Cambridge University Press, Cambridge (1999). MR 1744046 (2000j:52016)

5. De Loera, J., Rambau, J., Santos, F.: Triangulations: Structures and Algorithms. Springer, to appear

6. Gel'fand, I.M., Kapranov, M.M., Zelevinsky, A.V.: Discriminants, Resultants, and Multidimensional Determinants. Mathematics: Theory \& Applications. Birkhäuser, Boston (1994). MR 1264417 (95e:14045)

7. Grünbaum, B.: Convex Polytopes, 2nd edn. Graduate Texts in Mathematics, vol. 221. Springer, New York (2003). Prepared and with a preface by Volker Kaibel, Victor Klee and Günter M. Ziegler. MR 1976856 (2004b:52001)

8. Herrmann, S., Jensen, A., Joswig, M., Sturmfels, B.: How to draw tropical planes. Electron. J. Combin. 16(2), R6 (2009-2010)

9. Herrmann, S., Joswig, M.: Splitting polytopes. Münster J. Math. 1, 109-141 (2008)

10. Hirai, H.: A geometric study of the split decomposition. Discrete Comput. Geom. 36(2), 331-361 (2006). MR 2252108 (2007f:52025)

11. Shemer, I.: Neighborly polytopes. Israel J. Math. 43(4), 291-314 (1982). MR 693351 (84k:52008)

12. Ziegler, G.M.: Lectures on Polytopes. Graduate Texts in Mathematics, vol. 152. Springer, New York (1995). MR 1311028 (96a:52011) 